\title{
Research on the Prediction of Solar Energy Generation based on Measured Environmental Data
}

\author{
Guojing Zhang ${ }^{1}$, Xiaoying $\mathrm{Wang}^{1 *}$ and Zhihui $\mathrm{Du}^{2}$ \\ ${ }^{1}$ Department of Computer Technology and Applications, Qinghai University, \\ Xining, Qinghai, 810016, China \\ ${ }^{2}$ Tsinghua National Laboratory for Information Science and Technology, \\ Department of Computer Science and Technology, Tsinghua University,Beijing, \\ 100084, China \\ *Corresponding Author's Email: wxy_cta@qhu.edu.cn
}

\begin{abstract}
As a kind of renewable energy, solar power becomes more and more widely used as the power supply for large-scale datacenters to save the brown energy consumption and to reduce the overall cost. The prediction accuracy of solar energy generation becomes a fundamental issue in the research of how to efficiently manage the renewable energy resources. This paper explores the possible ways to predict the solar radiation intensity based on the assumption that it impacts the solar power generation proportionally. Through the analysis and research of photovoltaic power generation system, we explore the influence factors for solar radiation intensity, establish a relationship of solar radiation intensity and ambient temperature, time, humidity, wind speed in the forecasting model, and finally established the multivariate linear regression model and artificial neural network model. According to the two models, the environmental monitoring data measured at the Qinghai University are employed as the basis of the prediction of solar radiation intensity, and compared with the actual measurement data monitoring system. Experimental results show that, by using the BP neural network prediction model, the achieved accuracy is higher than other empirical model. The prediction method and good results provide a necessary foundation for future related research based on solar radiation values forecasts.
\end{abstract}

Keywords: solar radiation intensity; linear regression; artificial neural network

\section{Introduction}

Solar energy is one kind of the most potential renewable energy as a new mode of production for its cleanness and sufficient. Others have noted that over appropriate timescales and under ideal conditions the past predicts the future for both solar [1-3]. Nevertheless, due to the instability of photovoltaic power generation, it would have unpredictable impact on existing grid structure. Thus, if the solar radiation intensity of a given region could be accurately predicted, the generated solar power could be better utilized and bring greater benefits.

Facing these problems, this paper presents two possible prediction methods. For a variety of factors that affect the intensity of solar radiation, a multivariate linear regression model is first established, incorporating various factors such as solar radiation intensity and time, ambient temperature, humidity, wind speed and so on. Experimental results show that the multivariate linear regression model results in greater prediction errors, and the prediction results are instable. Similarly, based on these factors, the artificial neural network (ANN) model is also established and examined. The predicted 
results show that the ANN model is more effective, reliable and can achieve high prediction accuracy.

The rest of the paper is organized as follows. The related work is introduced in Section 2. Preparatory analysis about the solar radiation generation and related impacting factors is presented in Section 3. Then, the prediction models is established and introduced in Section 4. Comparison results of performance evaluation experiments are given in Section 5. In Section 6, we studied some cases and analyzed the errors using two different models under different scenarios. Finally, Section 7 concludes the whole paper.

\section{Related Work}

In this section, we discuss the related work in the area of solar energy prediction.

There are many predictive models of solar energy generation such as linear regression, radial basis function model, ARMA model[4], neural network model and so on. Photovoltaic power prediction method is divided into two categories: indirect and direct methods. Yunxue Song et al. [5] employed an indirect method, which used historical data of the light intensity through artificial neural network model to predict the intensity of solar radiation. PV output power is calculated by the light intensity, ambient temperature and other parameters. Yang Si et al. [6] adopted a direct method, which used historical data from photovoltaic power generation system, and established SVM and BP neural network model to predict the amount of PV power generation.

MELLIT et al. [7] used Radial Basis Function (RBF) networks in order to find a model for daily global solar radiation data from sunshine duration and air temperature. This methodology is considered suitable for prediction time series. The advantage of this model is able to predict and estimate a sequence of daily solar radiation data from only temperature air and sunshine duration, which are always available. Jianping Wang et al. [8] combined GA and WNN to construct the daily solar radiation prediction model. The advantage of their method is that the model can predict daily solar radiation well and it's an accurate and reliable method to predicate daily solar radiation. R.Yacef et al. [9] presents a comparative study between Bayesian Neural Network (BNN), classical Neural Network (NN) and empirical models for estimating the daily global solar irradiation (DGSR). Results show that the BNN performs better that other NN structures and empirical models.

Luis Mart1'n et al. [10] presented a comparison of statistical models applied on clearness index and lost component to forecast half daily values of solar irradiance for the next 3 days. The main advantage of the methodology exposed here is its simplicity to obtain predictions of solar irradiance. Ji Wu et al. [12] tested several models' performance in detrending the hourly solar radiation and found that the prediction of the hybrid model is quite good since it is stable and accurate. They also proposed a novel multi-model prediction framework for solar radiation time series [13], and find the advantage of MMF is that it models non-linear relationship of different pattern with different TDNN separately rather than simply using one TDNN to capture the general trend of whole series. BarisAksanli et al. [14] designed an adaptive data center job scheduler which utilizes short term prediction of solar and wind energy production.

\section{Preparatory Analysis}

This section describes the data sources used throughout the paper, analyzes the relationship between solar power generation and solar radiation intensity, and tries to give out some factors impacting the solar radiation intensity. 


\subsection{Data Sources}

The data used throughout this paper are measured on top of the Qinghai University Engineering Laboratory Building, with the longitude of $101^{\circ} 45^{\prime} 1.17^{\prime \prime} \mathrm{E}$ and the latitude of $36^{\circ} 43$ '35 .62" N. The building is located in eastern Qinghai-Tibet Plateau, and the ground is open without obstruction. The data are measured and collected by TMC-2ST, which is a recording instrument produced by DongGuan Greenlight Company Limited. The measurement error is 5\%. The recorded data contained the sun radiation intensity, temperature, weather conditions, and the exact date and time when measured. The collecting period starts from October 2nd, 2013 to October 22nd, 2013 and January 9th, 2014 to March 9th 2014, and over 90,000 pieces of data are recorded. The data are measured through 24 hours a day, and once in every 10 minutes.

\subsection{The Relationship between Solar Power Generation and Solar Radiation Intensity}

Here, we attempt to examine the calculation of the energy generated by photovoltaic equipment. A simple model of solar power generation can be presented as follows:

$$
P=N \bullet A_{p} \bullet \varphi \bullet \eta_{p} \bullet \eta_{D C}
$$

(1)

Where $N$ is the number of solar panels, $A_{p}$ is the surface area of each panel, $\varphi$ represents solar radiation intensity, $\eta_{p}$ represents the efficiency of the solar panel, and $\eta_{D C}$ represents the maximum power tracker solar array efficiency. Since the photovoltaic power generation is volatile and intermittent, $\varphi$ is usually of strong randomness. It might be affected by season, temperature, pressure, humidity, cloud and other factors. Therefore, from the equation it can be seen that, in the same environment daily solar power generation solar panel $N$, the surface area of each panel $A_{p}$, the efficiency of the solar panel $\eta_{p}$, the maximum power tracker solar array efficiency $\eta_{D C}$ are relatively stable, but only $\varphi$ might randomly changes. Thus, we need to focus on the study of solar radiation intensity $\varphi$, in order to capture the solar power variation.

Solar radiation intensity refers to the vertical projection unit time in earth's solar radiation energy per unit area. Therefore, solar radiation intensity directly affect the PV system, with a positive correlation. In summary, the solar intensity is proportional to the amount of solar radiation. The greater the intensity of solar radiation, the more the amount of solar power, and vice versa. Due to the limitations of the experimental equipment, the paper measured solar radiation intensity, ambient temperature, humidity, wind speed, time, and use these data to predict solar radiation intensity.

To accurately predict solar radiation intensity is the key issue of this paper. Here we present two possible prediction methods. According to the factors influencing the intensity of solar radiation, we established a multivariate linear regression model, considering factors including solar radiation intensity and time, ambient temperature, humidity, wind speed and other weather conditions. Experimental results show that the prediction error of multivariate linear regression model is relatively large, and the results are not stable. Similarly, based on these factors, we establish artificial neural network model, predicted results show that this model is effective, reliable and can achieve high prediction accuracy.

\subsection{Impacting Factors of Solar Radiation Intensity}

3.3.1 Impact of weather types:The intensity of solar radiation is uncertain, but is also strongly cyclical. Photovoltaic power generation system brings positive intensity values of solar radiation in the period of 8:00 A.M. to 6:00 P.M. each day. Figure 1 shows that solar radiation intensity variation from Oct.4th, 2013 to Oct.5th, 2013, measured by the same PV systems at the Qinghai University on two continuous sunny days. 
As can be seen from Fig.1, the solar radiation intensity of sunny days change regularly, which is time continuous and uniform, showing approximately of normal distribution trend. The effective intensity of solar radiation lasts from 8:00 A.M. to 6:00 P.M. The highest intensity of solar radiation occurs during 13:00-14:00, about $800 \mathrm{~W} / \mathrm{m} 2$. When the weather is sunny, it will have a good predictive of solar radiation intensity, and have a high prediction accuracy.

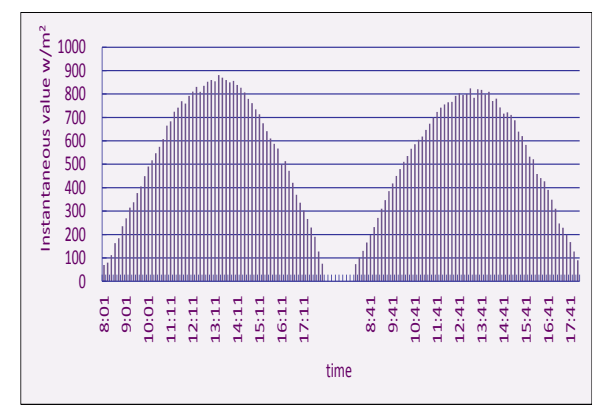

Figure 1. Solar Radiation Intensity of Two Continuous Sunny Days

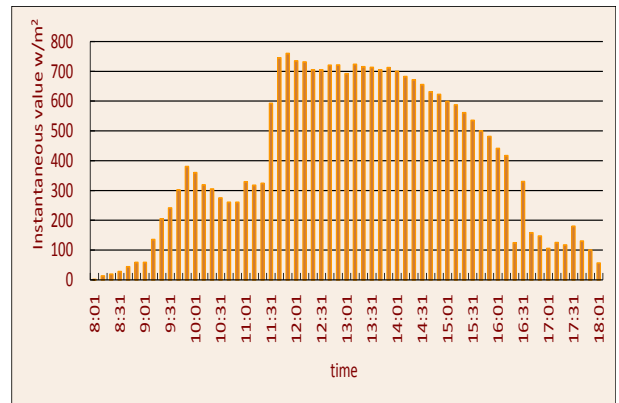

Figure 2. Cloudy to Sunny Solar RadiationIntensity Variation

The data in Fig.2 are measured at Qinghai University on Oct 13th, 2013, in order to analyze the variation of solar radiation intensity. As it can be seen, when the weather turns from sunny to cloudy becoming sunny, the trend of the solar radiation intensity variation is from low to high. At this time, if we don't consider the input variables to reflect the change of solar radiation intensity in time, the prediction of solar radiation intensity will be inaccurate. Therefore, we need to select the appropriate variables to reflect the changes of solar radiation intensity.

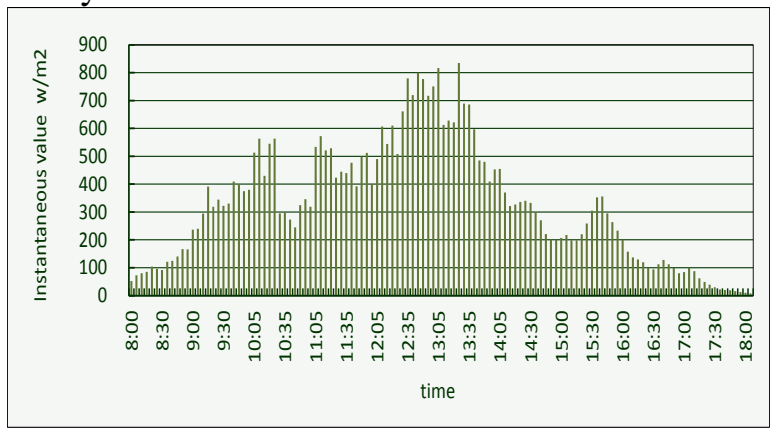

Figure 3. Solar Radiation Intensity Variation on a Cloudy Day

The data in Fig.3 are measured at Qinghai University on Oct.15th, 2013 in order to analyze the variation of solar radiation intensity. As it can be seen in Fig. 3, when the weather type of the day is cloudy, the variation of solar radiation intensity is not remarkably regular and randomly fluctuates. 
To sum up, when weather conditions of different days are similar, despite of the slight difference of the solar radiation intensity, the variation of solar radiation intensity will also be similar. If the weather types re not similar, the solar radiation intensity of different days will be greatly differentiated. Hence, the change of the solar radiation intensity is a non-stationary random process. Therefore, in order to improve the prediction accuracy, the weather types need to be classified, for example, sunny, cloudy, overcast and so on.

\section{Prediction Models based on Environmental Monitoring}

In this section, we present the two prediction models we designed and adopted for forecasting the solar energy radiation values.

\subsection{Multivariate Linear Regression Model}

Multivariate linear regression forecasting method is a predicting method of linear relationship between a dependent variable and multiple dependent variables. The predictive model of multivariate linear regression analysis is as follows:

$$
y=\beta_{0}+\beta_{1} x_{1}+\beta_{2} x_{2}+\ldots .+\beta_{p} x_{p}+\varepsilon
$$

Where the unknown parameters $\beta_{0}, \beta_{1}, \beta_{2} \ldots \ldots \ldots \beta_{p}$ are called the regression coefficients. According to a set of observation $\left(x_{1}, x_{2}, x_{3} \ldots \ldots x_{i}\right), i=1,2, \ldots \ldots . n$, the estimated values of $\beta_{0}, \beta_{1}, \beta_{2} \ldots \ldots . \beta_{p}$ could be calculated, and then the multivariate linear regression equation could be obtained. In Eq. (2), $\varepsilon$ is a random error, and we often assume that:

$$
\left\{\begin{array}{c}
E(\varepsilon)=0 \\
\operatorname{Var}(\varepsilon)=\sigma^{2}
\end{array}\right.
$$

Then $\mathrm{E}(y)=\beta_{0}+\beta_{1} x_{1}+\beta_{2} x_{2}+\ldots . \beta_{p} x_{p}$ is called the theoretical regression equation.

\subsection{Artificial Neural Network Modeling}

BP neural networkwas first proposed by Rumelhart and McCellandin 1986 [14], which is one of the most successful and widely-used neural network. The structure diagram of BP neural network is as shown in Fig.4. The learning process consists of two stages: forward propagation and backward of error propagation. Lapeds and Farber first applied the artificial neural network onto prediction in 1987 [15]. The historical data of input and output variables should first be chosen to train the neural network, and then the connection weights and thresholds could be determine. Finally, the fitting of historical data could be performed.

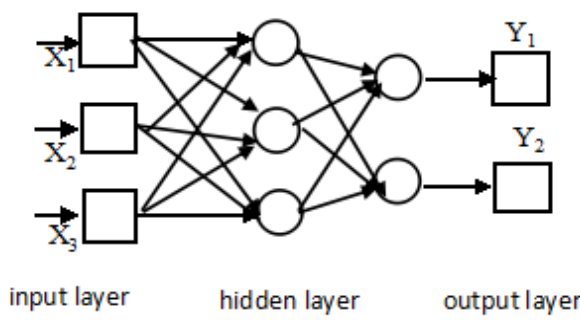

Figure 4. BP Neural Network Structure Diagram

\section{Performance Evaluation Experiments}

This section reports the performance evaluation results on the effectiveness of the two different prediction models. 


\subsection{Prediction Results of Multivariate Linear Regression Model}

5.1.1 Different weather conditions:After establishing the model, the data will be processed and unavailable data will be removed. The linear regression model is established using 542 sets of data measured from October 4th to October 12th, 2013. We explore the influence factors of solar radiation intensity, establish a relationship of solar radiation intensity and ambient temperature, time, humidity, wind speed between the forecasting model and established multivariate linear regression model. We use MATLab to establish a multivariate linear regression model based on the data measured from October 4 th to October 10th, and solved the multiple linear regression equation. The multivariate linear regression model solved by MATLab is as follows:

$$
y=2408.9+275.8 x_{1}+27.1 x_{2}-13.4 x_{3}-193 x_{4}(9)
$$

According to the prediction equation of multivariate linear regression model, the intensity of solar radiation on October 11th is predicted based on the weather data such as environmental temperature, humidity, wind speed, and so on. Fig.5 is the prediction residual plot, and Fig. 6 is the comparison chart of measured values and predicted values of the solar radiation intensity on a sunny day.

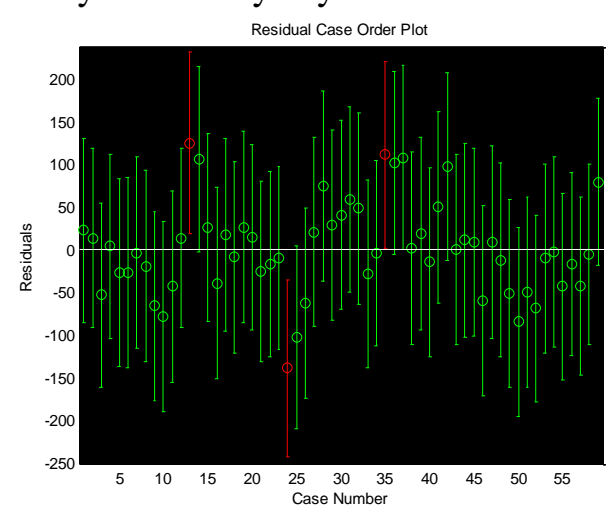

Figure5. Residual Plot (Sunny Day)

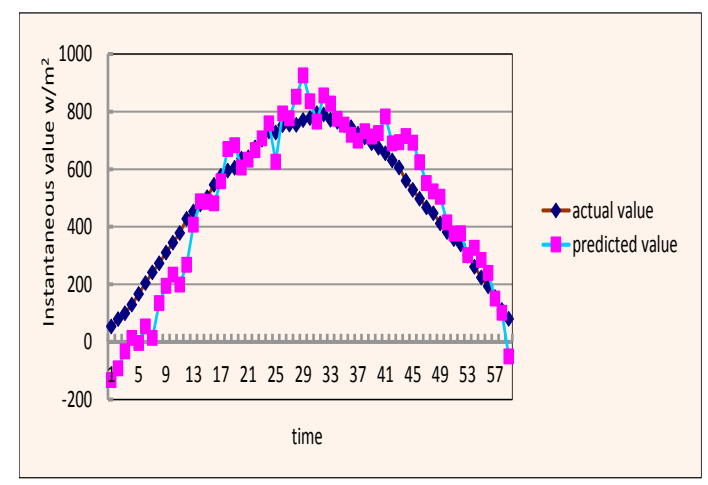

Figure 6. Comparison of the Predicted Valuesand Measured Values of the Solar RadiationIntensity (Sunny Day)

The linear regression model of sunny days shows that the most accurate prediction is usually achieved by using the model calculated from the previous day, with the smallest error. However, if the weather turns from sunny to cloudy, the prediction results have to be verified. On a cloudy day, the variation of solar radiation intensity is not obvious and exhibits random fluctuation. 
We used 240 sets of data, measured in October 15th to October 16th, 2013, for the regression analysis. We tried to predict the solar radiation value on Oct. 16th based on the measured data on Oct. 15th. The weather is cloudy on both two days. We used the environmental temperature, humidity, and wind speed as variables, and the intensity of solar radiation as the dependent variable, to establish a multivariate linear regression model. The multivariate linear regression model solved by MATLab is as follows:

$$
y=3852.4-1.7 x_{1}+44.5 x_{2}-31.9 x_{3}-149.6 x_{4}(10)
$$

According to the prediction equation of multivariate linear regression model, the intensity of solar radiation on October 16th is predicted based on the weather data on the previous day such as environmental temperature, humidity, wind speed, and so on. Fig.7 is the prediction residual plot, and Fig. 8 is the comparison chart of measured values and predicted values of the solar radiation intensity on a cloudy day.

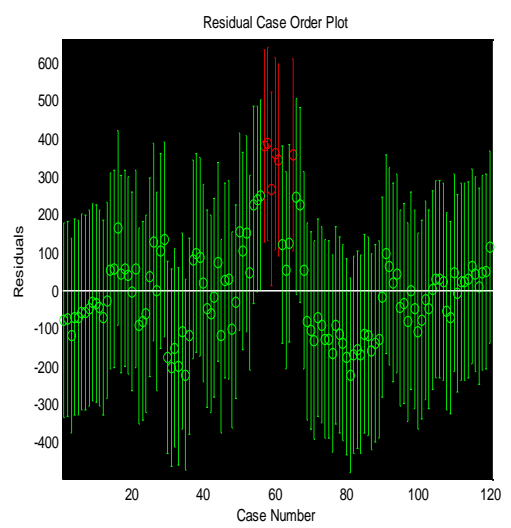

Figure 7. Residual Plot

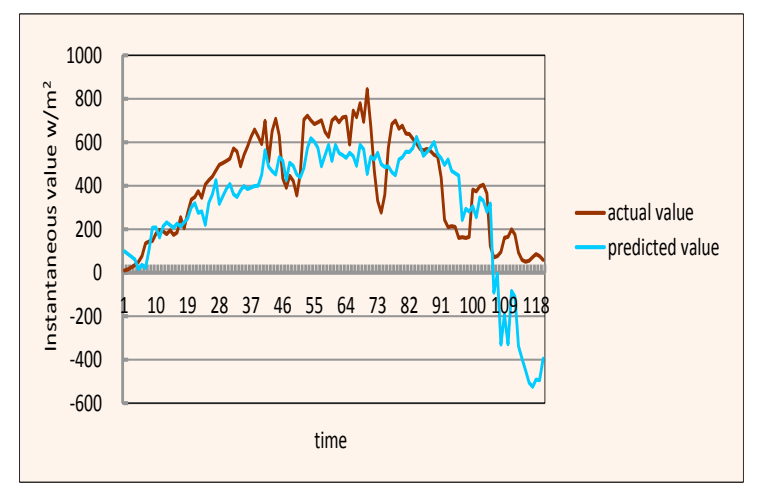

Fig. 8. Comparison of the Predicted Values and Measured Values of the Solar Radiation Intensity (Cloudy Day)

5.1.2 Comparison of Prediction Results and Parameter Estimation: For sunny days, we check the statistics of regression model according to the prediction results, where $R^{2}=0.9<1$ (The correlation coefficient $R^{2}$ closer to 1 means the regression equation more significant), $F=228.4$ (higher $F$ means the regression equation more significant), $p<0.0001$ ( $p$ is the possibility corresponding to $F$, which is the lower the better), $s^{2}=328.43\left(s^{2}\right.$ is the variance of the residuals, which is the lower the better). For cloudy days, we also check statistic of regression model according to the prediction results, where $R^{2}=0.8<1, F=49, p<0.0001$, and $\mathrm{s}^{2}=1774.6$.It can be observed that the predicted results of multivariate linear regression model show 
large error and low stability. The comparison table of parameter estimation as shown in Table 1:

Table 1. Parameter Estimation Comparison

\begin{tabular}{ccllc}
\hline weather & $R^{2}$ & $F$ & $p$ & $s^{2}$ \\
\hline Sunny & 0.9 & 228.4 & $\mathrm{p}<0.0001$ & 328.43 \\
Cloudy & 0.8 & 49 & $\mathrm{p}<0.0001$ & 1774.6 \\
\hline
\end{tabular}

We can see that the weather type have significant impact on forecasting effect when using multivariate linear regression equations to predict the intensity of solar radiation. The prediction is relatively accurate for sunny days, but inaccurate and unstable for cloudy days.

Table 2. Different Types of Errors of Contrast

\begin{tabular}{ccc}
\hline $\begin{array}{c}\text { Multiple linear regression } \\
\text { model }\end{array}$ & Cloudy & Sunny \\
\hline Minimum relative error & 10.2299 & 2.0174 \\
Maximum relative error & 596.5359 & 228.3199 \\
Average relative error & 93.9877 & 74.9203 \\
MPE & 12.7485 & -18.5143 \\
MBE & -71.9854 & -5.6369 \\
RMSE & 122.7010 & 94.8996 \\
$\mathrm{R}^{2}$ & 0.8192 & 0.9229 \\
\hline
\end{tabular}

As it can be seen from Table 2, the predictive effect is not very ideal using multivariate linear regression model to forecast. The model is easy to use, but the prediction accuracy is low, with large maximum error, mean error, MBE, and RMSE. Therefore, prediction for sunny days leads to much better results for cloudy days. Due to the great error of the multivariate linear regression model, it cannot meet the requirement for the prediction accuracy and efficiency. In the following section, we further exploit the BP neural network model which can deal with the nonlinear results, and thus can make the error smaller.

\subsection{Artificial Neural Network Model}

5.2.1 Comparison of Prediction Results with Variable Nodes: Artificial neural network model requires a lot of data as the historical basis, divided into a training set and a data set. Neural network method has great advantage of solving nonlinear problems. Main prediction methods include Adaptive Neural Fuzzy Inference Scheme (ANFIS) [16], Radial Basis Function Networks (RBFN) [17], and Recurrent Back-Propagation Network (RBPN). In this paper, a BP neural network model is established upon different weather conditions. For sunny days, we use 485 groups of data from October 2th to October 12th in total as test samples, including the environmental temperature, humidity, wind speed, and time as four input variables, the solar radiation intensity as an output variable. 366 of 485 groups of data are chosen from input and output data as training samples, 120 groups as the validation sample, and then the artificial neural network model is established.

The number of the hidden layer neurons in the artificial neural network model also has some impact on the prediction results. The calculation formula for the number of hidden layer is $n=2 m+1$, where $m$ denotes the number of neurons in the input layer, and $\mathrm{n}$ denotes the number of neurons in the hidden layer. We assume that $n$ is 3 and establish the BP neural network. The training error is as shown in Fig.9.Taking the prediction results of two days as the validation samples, the comparison of actual values of solar radiation intensity and the predicted values is shown in Fig. 10.From the prediction results we can see that the model can achieve high efficiency and high predictive precision. 


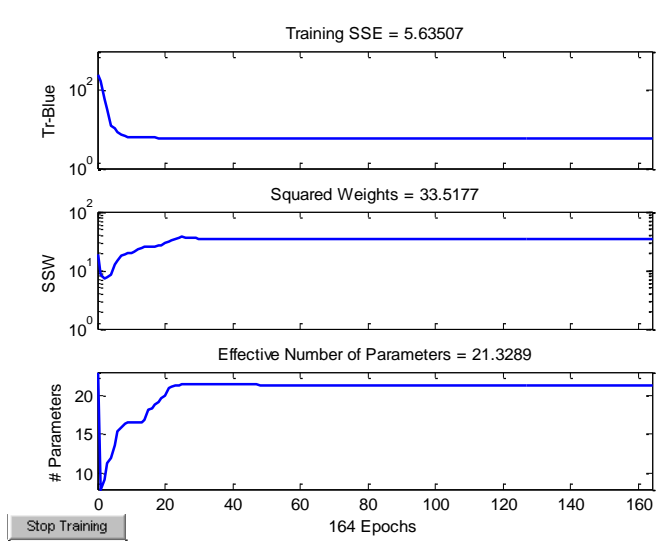

Figure9. Training Error of the Neural Network

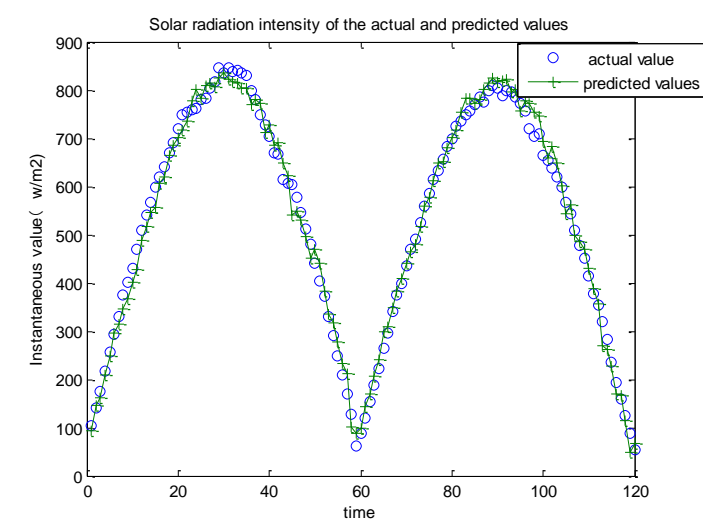

Figure 10. Comparison of the PredictedValues and Actual Values of Solar Radiation Intensity

Based on the above results, we can explore further to find out a better fitting. In this network, the number of neurons in input layer and output layer are respectively 4 and 1 . According to the calculation formula of hidden layer, we set the number of neurons in the hidden layer of network to 2 9. Therefore, according to the sample data previously used, we designed BP neural network with variable number of hidden layer neurons. Through the error comparison, we can determine the optimal number of neurons in the hidden layer. The values of calculated results are as shown in Table 3.We can find that the error is smallest when the number of hidden layer neurons is set to 8 .

Table 3. Error of Variable Number of Different Neurons in the Hidden Layer

\begin{tabular}{lcccccccc}
\hline $\begin{array}{l}\text { The number of } \\
\text { neurons }\end{array}$ & 2 & 3 & 4 & 5 & 6 & 7 & 8 & 9 \\
\hline Network error & 1091 & 1012 & 937 & 914 & 889 & 871 & 814 & 904 \\
\hline
\end{tabular}

When the number of hidden layer neurons $\mathrm{n}$ is 8 , the training error is as shown in Fig.11. The comparison of actual values and predicted values of solar radiation intensity is as shown in Fig. 12. 


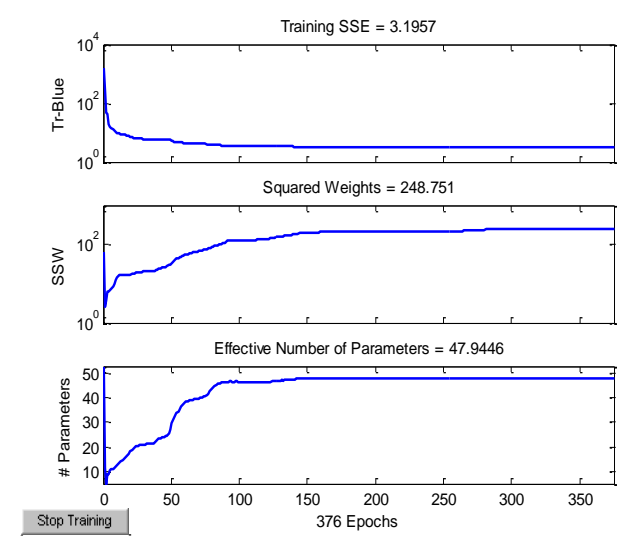

Figure 11. Neural Network Training Error $(n=8)$

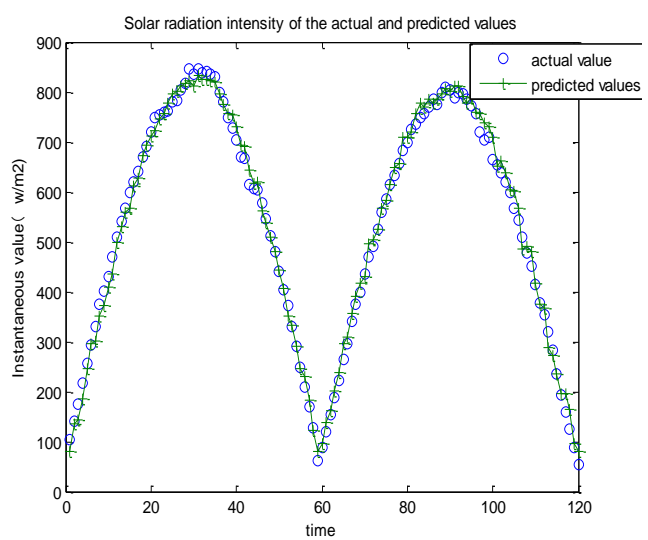

Figure 12. Comparison of the Predicted Values and Actual Values of Solar Radiation Intensity $(n=8)$

As seen from Fig. 12, using artificial neural network for prediction could achieve high accuracy, with good approximation of the functions. Fig. 13 shows the relative error percentage of the solar radiation intensity when $n=3$ and $n=8$. As it can be seen from the figure, in both cases, most of the relative errors are less than $10 \%$, except some special cases.
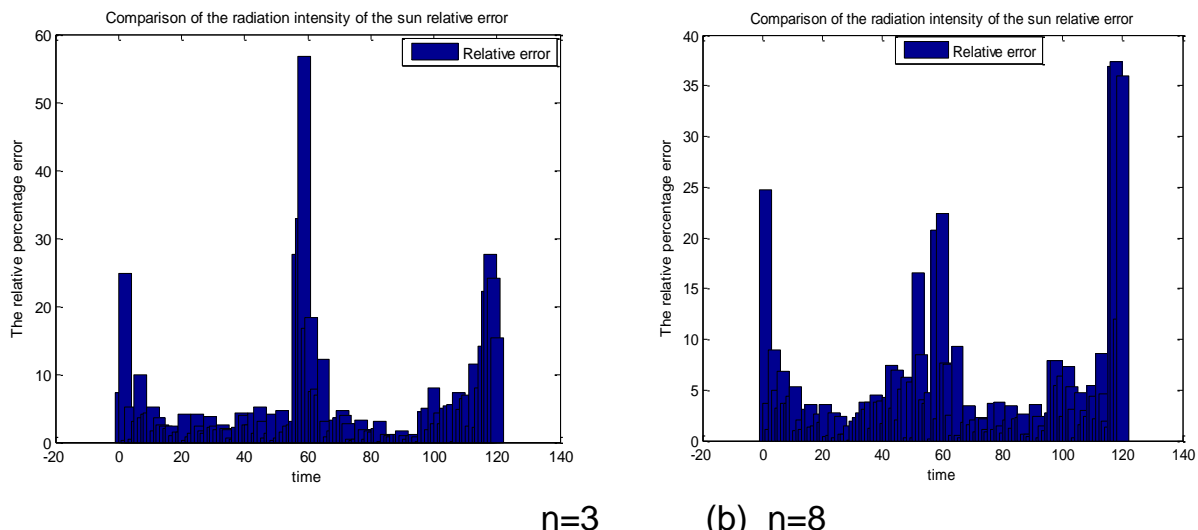

$n=3$

(b) $n=8$

Figure 13. Comparison Figure of the Radiation Intensity of the Solar Relative Error 
5.2.2 Prediction Results of Different Training Function: Different network training function can also impact the prediction results. In order to achieve high accuracy, the network training function should be deliberately selected in addition to adjusting the number of hidden layer.

Here, we use a series of functions, such as trainbr, trainlm, traingdm, traingdx, trainbfg and so on, to predict the intensity of solar radiation. After experiments, we found that trainbr leads to the best results. In order to verify the prediction efficiency of trainbr function, here we take two training functions as examples, including trainlm and trainbr. The use of trainlm network training function are as follows:

net=newff(PR,[1,1],\{ 'tansig','purelin'\},'trainlm','learngdm','mse');

Fig.15 illustrates the comparison of prediction results using different training functions. As it can be seen from the graph, the result curve of trainbr is more smooth, and closer to the curve of actual values. However, the results by using trainlm function contains much more errors. So it can be seen from Fig. 15, using trainbr training function is better than using trainlm function during the prediction process.

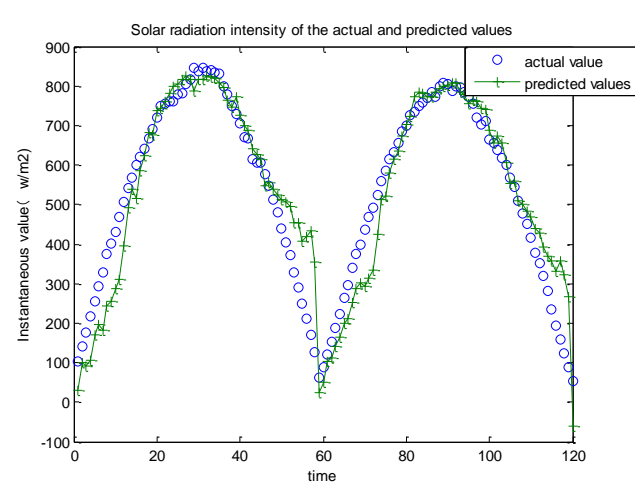

(1)The Training Function for Trainlm

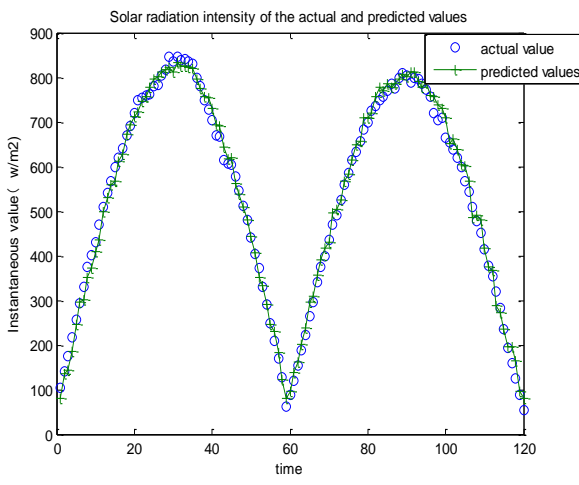

(2) The Training Function for Trainbr

Figure 15. The Prediction Results of Different Training Functions

Fig.16 shows the statistics of the relative percentage error when using the training function trainbr and trainlm to predict the solar radiation intensity. As can be seen from the figures, when using the training function trainbr, the relative percentage errors could be controlled within $40 \%$, and most of the errors are less than $10 \%$, which basically meets the requirements proposed in "Twelfth Five Year Plan for Qinghai Province" [7]. In contrast, the relative errors of trainlm are too large, some of which even above $200 \%$, so that this function is not suitable to use for prediction.
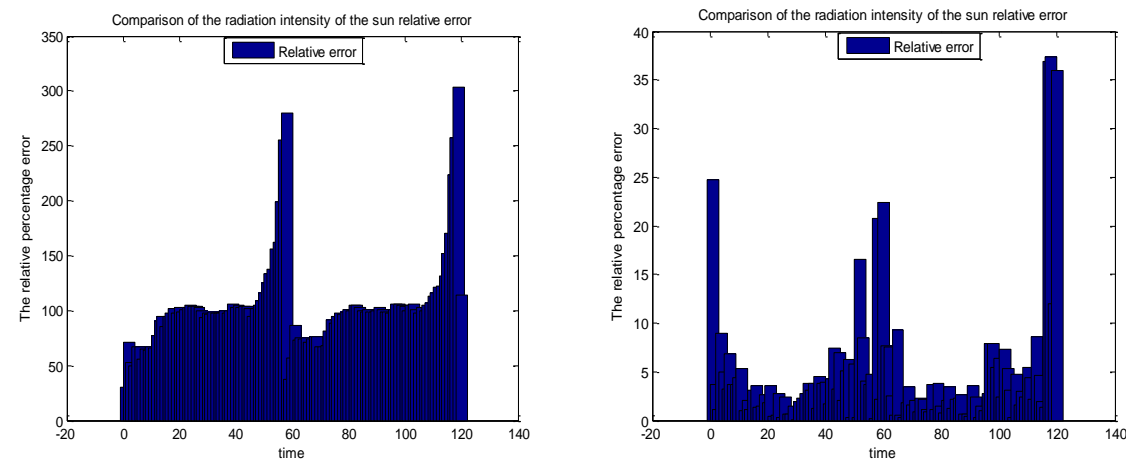

(1)The Training Function for Trainlm(2) The Training Function for Trainbr

Figure 16. Relative Error of Different Training Function Comparison Chart 
We also attempt to explore the reason why the trainbr function results in faster convergence speed and higher precision fitting. The trainbr function uses the Bayesia regularization method, and can improve the generalization ability of neural network training by correcting performance functions, and thus can prevent "excessive training". Using trainbr training function in the BP neural network is superior to trainlm, traingdm, and other functions in our scenario. Only a few hundred times training can make the network convergent, and the optimal network weights and thresholds can be found.

5.2.3 Prediction Results under Different Weather Conditions: For sunny days, we select the hidden layer number $\mathrm{n}=8$, and the prediction results are shown as Fig. 12, For cloudy days, we selected 362 groups of data as the test samples, from October 12th to October 16th, including the environmental temperature, humidity, wind speed, and time as four input variables, the solar radiation intensity as an output variable. 242 groups of data are chosen from input and output data as training samples, 120 groups as the validation sample, and then the artificial neural network model is established.

Fig.17 shows that comparison of prediction results for sunny and cloudy days. We can see that, on sunny days, the change of the intensity of solar radiation has a certain regularity, and thus the prediction results show high efficiency and good stability. On cloudy days, the historical solar radiation intensity varies greatly, with poor regularity, so that the forecast effect is worse than on sunny days.

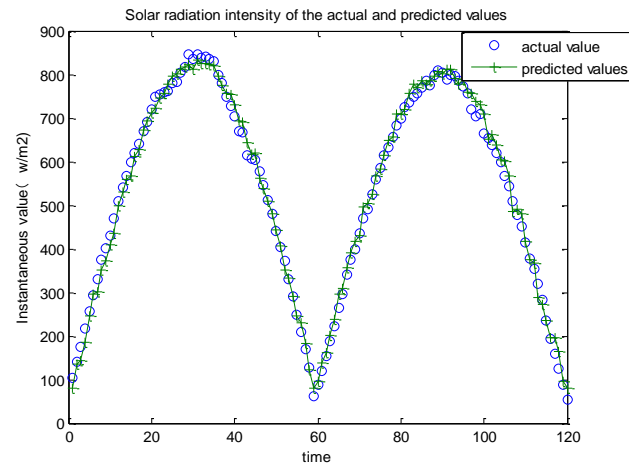

(1) Day Type: Sunny

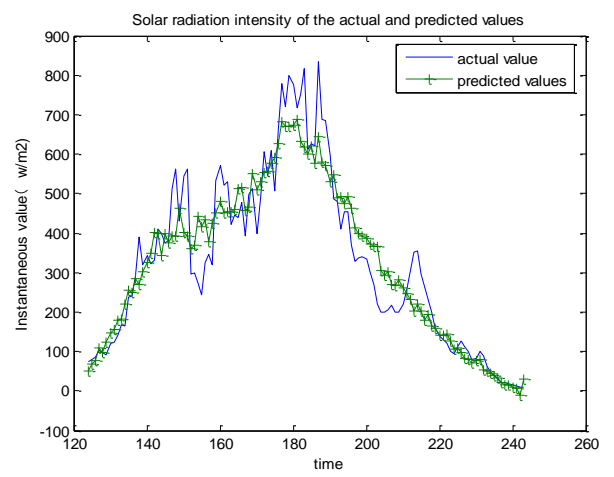

(2) Day Type: Cloudy

\section{Figure 17. The Predicted Value and Actual Values of Solar Radiation Intensity under Different Weather Conditions}

Fig.18 shows the statistics the relative error percentage of solar radiation intensity for sunny and cloudy days. As can be seen from the figure, for sunny days, the relative percentage error is within $16 \%$, and most of the error is less than $10 \%$. For cloudy days, the relative percentage error is more lager. Most of the error is less than 50\%, some even reached $200 \%$. On sunny days, since the weather changes smoothly, the prediction effect is very good. However, on cloudy days, since the weather varies unregularly, the forecast effect drops, but most errors still can be controlled under a certain level. 


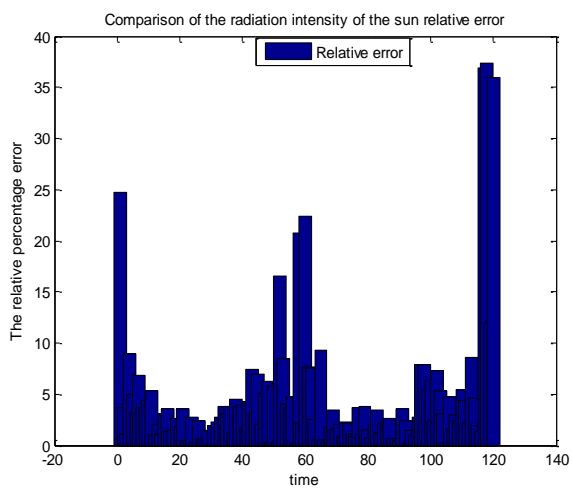

(1) Day Type: Sunny

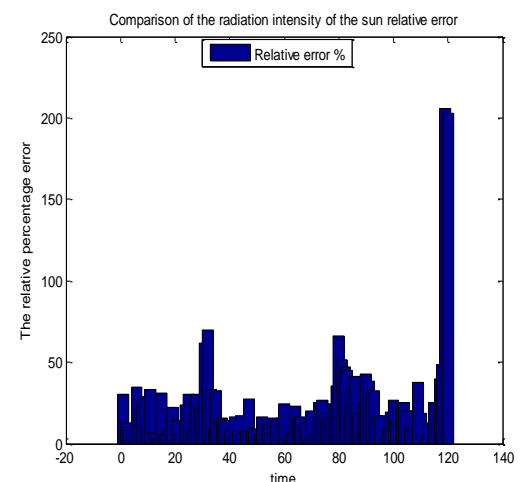

(2) Day Type: Cloudy

Figure18. Different Weather Types of Relative Error Comparison Chart

\section{Case Study}

\subsection{Comparison of the Two Models}

In this paper, we try to predict the solar radiation intensity using multivariate linear regression model and BP neural network model. In order to verify the validity of the models, we use multivariate regression model and also select photovoltaic output data and meteorological data as samples to train the neural network, comparing prediction results of two different models. The two models are employed to predict solar radiation intensity on October 5th (sunny) and October 15th (cloudy). Fig.19 is the comparison of different prediction models for solar radiation intensity of the cloudy day.

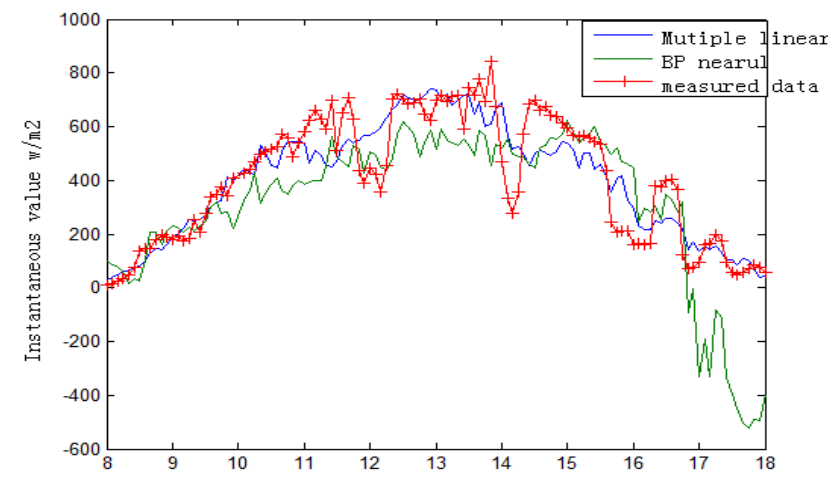

\section{Figure 19. Comparison of Different Prediction Models for Solar Radiation} Intensity (Cloudy)

As it can be seen in Fig.19, the change trends of the forecasting results of artificial neural network and the multivariate linear regression model are generally consistent. The prediction accuracy drop badly after 16:00 when using multivariate linear regression model. The reason is that on cloudy days, the intensity of solar radiation change a lot, leading to instable prediction results. Fig.20 is the comparison of prediction errors using two kinds of models. As can be seen from the figure, BP neural network model behaves better than the multivariate linear regression model. 


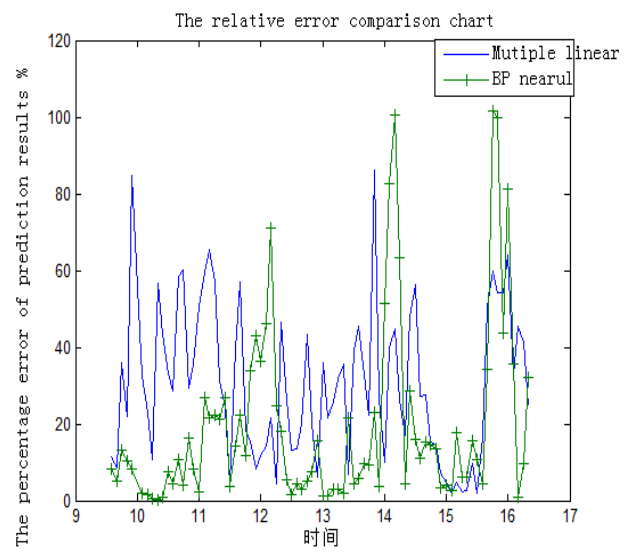

Figure20. Comparison of the Relative Errors (Cloudy)

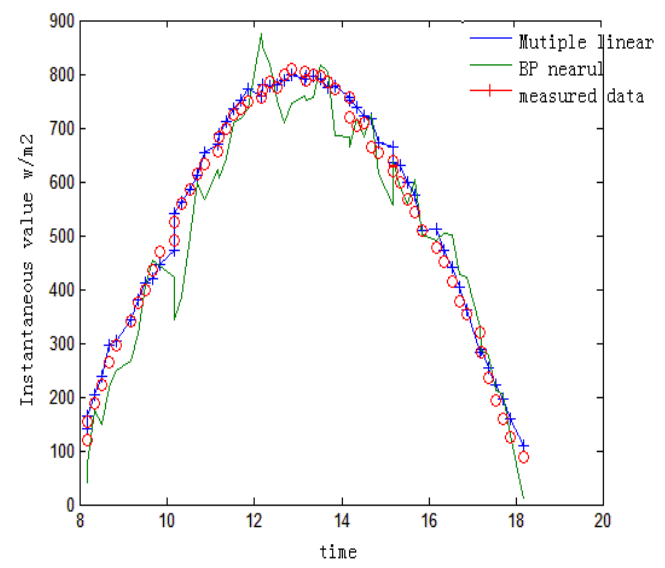

Figure 21. Comparison of Different Prediction Models for Solar Radiation Intensity (Sunny)

Fig.21 is the comparison of different prediction models for solar radiation intensity on sunny days. From the figure we can see that the prediction of the artificial neural network and multivariate linear regression model both can achieve higher accuracy than on cloudy days. Comparatively, the BP neural network model behaves better than multivariate linear regression model. The curve of the BP neural network model is smoother than the multivariate linear regression model, which shows the advantage of the artificial neural network. Fig. 22 is the comparison of the prediction error of the two kinds of different models. As can be seen from the figure, the errors of both models are smaller, where most of the errors of BP neural network can be controlled in 20\%. In contrast, the errors of multivariate linear regression model show obvious fluctuation. 


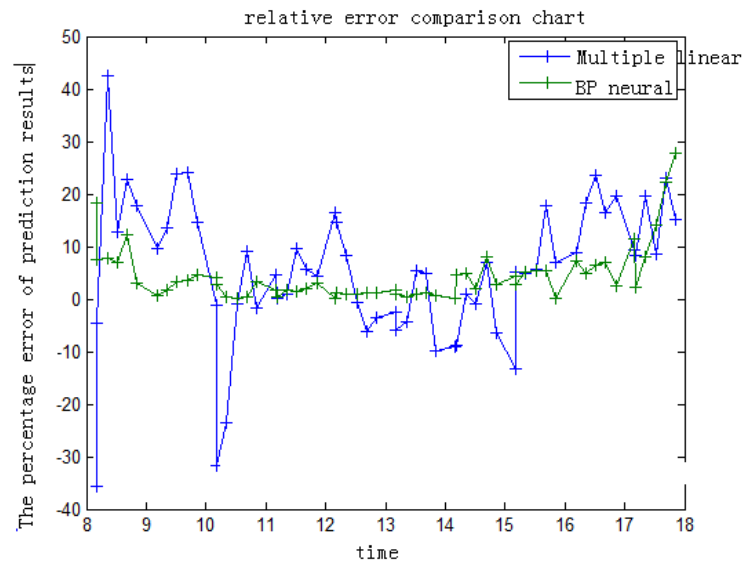

Figure 22.The Relative Error Comparison Chart (Sunny)

\subsection{Error Analysis for Two Models in Different Weather}

Table 4 shows the statistics error analysis of the two models in different weather. By calculating the relative error, the average percentage error, MPE, MBE, and RMSE, we can analyze the predicted effect of solar radiation intensity using different prediction methods under different weather.

Table 4. Error Analysis of Different Methods in Different Weather

\begin{tabular}{ccccc}
\hline Error analysis & $\begin{array}{c}\text { BP neural } \\
\text { network } \\
\text { (cloudy) }\end{array}$ & $\begin{array}{c}\text { BP neural } \\
\text { network } \\
\text { (sunny) }\end{array}$ & $\begin{array}{c}\text { Multivariate linear } \\
\text { regression (cloudy) }\end{array}$ & $\begin{array}{c}\text { Multivariate linear } \\
\text { regression (sunny) }\end{array}$ \\
\hline Minimum relative error & 0.6248 & 0.1104 & 10.2299 & 2.0174 \\
Maximum relative error & 206.032 & 37.3360 & 596.5359 & 228.3199 \\
Average relative error & 21.679 & 4.5813 & 93.9877 & 74.9203 \\
MPE & & & & -18.5143 \\
MBE & 1.7653 & 1.5537 & 12.7485 & -5.6369 \\
RMSE & 2.7643 & 3.7693 & -71.9854 & 94.8996 \\
$\mathrm{R}^{2}$ & 72.8662 & 18.0158 & 122.7010 & 0.9229 \\
\hline
\end{tabular}

The experimental data are measured at the Qinghai University from Oct. 1st, 2013 to Oct.21st, 2013. Based on these data, we established the multivariate linear regression model and artificial neural network model. Table 4 calculates all kinds of errors in different weather using different prediction methods. We can see from the table that the model of BP neural network prediction is better than multivariate linear regression model and the errors of BP neural network model could be controlled within 20\%. Furthermore, the goodness degree reached 0.9943 on cloudy days, very close to optimal results.

Table 5. Error Comparison of Different Nodes under Different Training Functions

\begin{tabular}{cccccccc}
\hline $\begin{array}{c}\text { Artificial } \\
\text { neural } \\
\text { network(sunny } \\
\text { ) }\end{array}$ & $\begin{array}{c}\text { Minimum } \\
\text { relative } \\
\text { error }\end{array}$ & $\begin{array}{c}\text { The } \\
\text { maximum } \\
\text { relative } \\
\text { error }\end{array}$ & $\begin{array}{c}\text { The } \\
\text { average } \\
\text { relative } \\
\text { error }\end{array}$ & MPE & MBE & RMSE & $\mathrm{R}^{2}$ \\
\hline trainlm $(\mathrm{n}=3)$ & 82.9439 & 341.6791 & 112.477 & 12.4768 & 5.6669 & 85.711 & 0.8803 \\
trainbr $(\mathrm{n}=3)$ & 0.0465 & 56.7479 & 5.0449 & 0.6969 & 6.0842 & 22.917 & 0.9904 \\
trainlm $(\mathrm{n}=8)$ & 0.0481 & 214.076 & 22.8665 & 2.6402 & 6.0842 & 19.631 & 0.9935 \\
trainbr $(\mathrm{n}=8)$ & 0.1104 & 37.3360 & 4.5813 & 1.5537 & 3.7693 & 18.016 & 0.9943 \\
\hline
\end{tabular}


Table 5summarizes the relative error statistics of different nodes and different training functions, including the average percentage error, MPE, MBE, and RMSE.As can be seen from the table, when using the artificial neural network, the number of nodes and network parameters have great impact on the prediction effect. When using trainbr as the training function and set $\mathrm{n}$ to 8 , the prediction can achieve best results according to the goodness of the fitting, which is closest to 1 . With the same number of nodes, there are big differences between the errors using different training functions. Using the same training function with different number of nodes, the differences of relative error, MPE,MBE, and RMSE are much smaller. Thus, the number of nodes doesn't have significant impact on the prediction effect but the choice of proper training function is more important. We should first choose a suitable training function, and continuously adjust the parameters, to achieve the optimal prediction effect.

\section{Conclusion}

In this paper, we first analyze the relationship between the solar radiation intensity and the amount of solar power, and find that the amount of solar power generation and solar radiation intensity is proportional. Through the proportional relation analysis of solar power generation and solar radiation intensity, we established the prediction model of the solar radiation intensity based on environmental factors such as temperature, time, humidity, wind speed and so on.

Then, we analyze the solar power generation forecasting principle and its impact factors and the variation of solar radiation intensity in different weather conditions through the study of historical data. The prediction results show that the prediction performs better for sunny days but worse for cloudy days. In order to improve the prediction accuracy, photovoltaic historical data should be classified based on the characteristics of the weather condition. Different seasons also have impact on solar radiation intensity variation. The intensity of solar radiation in autumn is relatively stable, but the variation of solar radiation intensity in winter is not the regular. Thus, weather and seasonal factors will lead to prediction deviation. Furthermore, we establish two prediction models of the solar radiation intensity respectively based on multivariate linear regression model and BP neural network, with the history of the power generation data as samples for training. Two kinds of models are both used to predict the solar radiation intensity and the results are compared. Experimental results show that BP neural network is better than the multivariate linear regression model, which is simple and fast but brings much bigger error. When using BP neural network prediction model, it behaves best when dealing with the prediction of sunny days, and the accuracy is higher than other empirical model. The good prediction results is meaningful for the future work on solar-energyrelated research fields. By comparing the prediction results under different weather conditions and using different methods and analyzing the errors, we find that the multivariate linear regression model is relatively simple, but the errors are significant. In contrast, the BP neural network model can achieve much better results.

However, in this paper we only predict the intensity of solar radiation, but not the amount of solar power generation. Moreover, there are still many problems such as the over-fitting phenomenon when using BP neural network. We are planning to do further optimization of the BP neural network and exploit other factors in order to improve the accuracy of solar radiation prediction under complex weather conditions.

\section{Acknowledgement}

This paper is supported in part by National Natural Science Foundation of China (No. 61363019, No. 61272087 and No.61073008), National Natural Science Foundation of Qinghai Province (No. 2014-ZJ-718, No. 2013-Z-930Q, No. 2014-ZJ-941Q), and Beijing Natural Science Foundation (No. 4082016 and No. 4122039). 


\section{References}

[1] J. Apt and A. Curtright, "The Spectrum of Power from Utility-scale Wind Farms and Solar Photovoltaic", Carnegie Mellon Electricity Industry Center, Technical Report CEIC, August (2004).

[2] P. Corke, P. Valencia, P. Sikka, T. Wark and L. Overs, "Long-durationSolar-powered Wireless Sensor Networks", EmNets, (2007).

[3] A. Kansal, D. Potter and M. Srivastava, "Performance Aware Tasking For Environmentally Powered Sensor Networks", SIGMETRICS Performance Evaluation Review, (2004).

[4] A. M. Muñoz, J. J. G. de la Rosa, R. Posadillo and V. Pallarés, "Short term forecasting of solar radiation", IEEE, (2008), pp. 1537-2541.

[5] Y. X. Song and K. X. Zhang, "Research on aeroengine performance parameters forecast based on multiple linear regression forecasting method", Journal of Aerospace Power, vol. 24, no. 2, (2009), pp. $1000-8055$.

[6] S. I. Yang, "Prediction method of ground receiving solar radiation power based on pattern matching", Journal of Qinghai University, vol. 30, no. 1, (2012), pp. 31-34.

[7] A. Mellit, M. Benghanem and M .Bendekhis, Artificial Neural Network Model for Prediction Solar Radiation Data: Application for Sizing Stand-alone Photovoltaic Power System.

[8] J. P. Wang, L. X. Yun, G. Z. Chen and X. B. Xu, "Daily Solar Radiation Prediction Based on Genetic Algorithm Optimization of Wavelet Neural Network".

[9] R. Yacef, M. Benghanem and A. Mellit, "Prediction of daily global solar irradiation data using Bayesian neural network: A comparative study", Renewable Energy, vol. 48, (2012), pp. 146-154.

[10] L. Martı'n, L. F. Zarzalejo, J. Polo, A. Navarro, R. Marchante and M. Cony, "Prediction of global solar irradiance based on time series analysis:Application to solar thermal power plants energy production planning", Solar Energy, vol. 84, (2010), pp. 1772-1781.

[11] J. Wu and C. K. Chan, "Prediction of hourly solar radiation using a novel hybrid model of ARMA and TDNN", Solar Energy, vol. 85, (2011), pp. 808-817.

[12] J. Wu and C. K. Chan, "Prediction of hourly solar radiation with multi-model framework".

[13] B. Aksanli, J. Venkatesh, L. Y. Zhang and T. Rosing, "Utilizing Green Energy Prediction to Schedule Mixed Batch and Service Jobs in Data Centers", Computer Science and Engineering Dept. (CSE) University of California.

[14] D. E. Rumelhart, G. E. Hinton and R. J. Williams, "Learning internal representations by error propagation", Parallel Distributed Processing: exploration in microstructure of cognition I, Cambridge: MIT Press, (1985), pp. 318-362.

[15] L. A. Farber, "Nonlinear signal processing using neural networks: prediction and system modeling", Technical Report LA-UR-87-2662, Los Alamos National Laboratory, (1987).

[16] A. Mellit, A. H. Arab, N. Khorissi and H. Salhi, "An ANFIS-based Forecasting for Solar Radiation Data from Sunshine Duration and Ambient Temperature", Power Engineering Society General Meeting, IEEE, (2007), pp. 1-6.

[17] A. Mellit, M. Benghanem and M. Bendekhis, "Artificial Neural Network Model for Prediction Solar Radiation Data: Application for Sizing Stand-alone Photovoltaic Power System", Power Engineering Society General Meeting, IEEE, (2005), pp. 40-44.

\section{Authors}

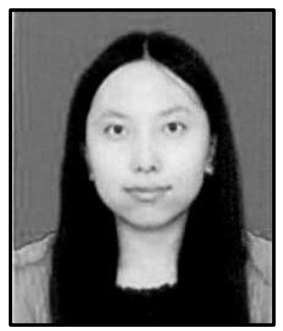

GuojingZhang, she receivedher bachelor's degreeinJuly 2014fromQinghai University. Since 2014 shehas beena graduate studentatQinghai University, she obtained excellent graduation thesis of computer system in 2014. Her research interests are high performance computing. 
International Journal of $u-$ and e- Service, Science and Technology Vol.8, No.5 (2015) 